\title{
Resveratrol Attenuates Nicotine-mediated Oxidative Injury by Inducing Manganese Superoxide Dismutase in Renal Proximal Tubule Cells
}

\author{
SAMUEL HALL, MEHUL DIXIT and ISTVAN ARANY \\ Department of Pediatrics, Division of Pediatric Nephrology, \\ University of Mississippi Medical Center, Jackson, MS, U.S.A.
}

\begin{abstract}
Background/Aim: Nicotine (NIC) exposure - via smoking and the increasingly popular E-cigarettes-increases oxidative stress and hence, renal risk in smokers. Resveratrol (RES) may help ameliorate this risk by mounting antioxidant responses in the kidney. Materials and Methods: Renal proximal tubule cells (NRK52E) were treated with vehicle or $20 \mu \mathrm{M}$ RES prior to treatment with $200 \mu \mathrm{M}$ NIC and generation of reactive oxygen species (ROS) as well as cell viability was determined. RES-induced antioxidant responses were determined in reporter luciferase assays. Gene silencing was used to determine mechanism of RES action. Results: RES protected NRK52E cells from NICinduced oxidative injury. RES activated the promoter of the anti-oxidant manganese superoxide dismutase (MnSOD) gene via activation of the forkhead box $O$ (FoxO3a) transcription factor. Silencing of MnSOD abolished the protective effects of RES on NIC-associated oxidative injury. Conclusion: RES may provide protection to the kidney from the adverse effects of NIC in smokers.
\end{abstract}

Smoking -through its nicotine (NIC) content (1) - is an important risk factor in progression of kidney diseases (2). Increasing popularity of E-cigarettes $(3,4)$ could further increase this risk as they are believed to be safe alternatives to tobacco smoking (3). While the best remedy would be to stop smoking, many smokers refuse to quit (5). Hence, alternative means may be needed to ameliorate adverse

This article is freely accessible online.

Correspondence to: Istvan Arany, Department of Pediatrics, Division of Pediatric Nephrology, University of Mississippi Medical Center, Jackson, MS, U.S.A. Tel: +1 6018159464, Fax: +1 6019845981, e-mail: iarany@umc.edu

Key Words: Nicotine, renal risk, oxidative stress, resveratrol, FoxO, MnSOD smoking. effects of smoking/NIC-exposure on the kidney. Since smoking/NIC exposure increases oxidative stress in the kidney $(6,7)$ and in cultured renal proximal tubule cells $(7$, 8 ), antioxidants may fulfill this protective role. Some naturally-occurring compounds, such as resveratrol (RES), have shown antioxidant capacity (9) and protective effects of RES against smoking-associated renal tubular damage have been shown (10). Regardless, the mechanism by which RES protects the kidney is virtually unknown.

Antioxidant properties of RES are associated with its ability to induce expression of antioxidant genes including manganese superoxide dismutase (MnSOD) $(11,12)$. One possible mechanism by which RES upregulates MnSOD is activation of the transcription factor cAMP responsive element binding protein (CREB) (13) or Forkhead box O (FoxO3a) (11). However, this effect of RES on the kidney under NIC exposure is unknown.

Accordingly, the aim of this study was to determine whether (i) RES protects renal proximal tubule cells from NIC-mediated oxidative injury and (ii) the protective effects of RES due to activation of the CREB/FoxO/MnSOD pathway.

\section{Materials and Methods}

Cell culture and treatment. The rat proximal tubule cell line (NRK52E) was maintained in $5 \% \mathrm{CO}_{2}$ at $37^{\circ} \mathrm{C}$ in DMEM with $10 \%$ FBS as described elsewhere (14). Some cultures were pre-treated with $20 \mu \mathrm{M}$ resveratrol (RES, Selleckchem, Houston, TX, USA) or vehicle (DMSO) prior to treatment with $200 \mu \mathrm{M}$ nicotine (NIC; Sigma-Aldrich, St. Louis, MOS, USA)

Determination of reactive oxygen species (ROS) production. Intracellular generation of ROS was determined by fluorescent oxidant-sensitive 2', 7'-dichlorofluorescein-diacetate dye (DCFDA; Life Technologies, Grand Island, NY, USA). Briefly, cells grown in T25 flasks and treated with $20 \mu \mathrm{M}$ RES or vehicle overnight were collected, counted and loaded with $100 \mu \mathrm{M}$ DCFDA for $30 \mathrm{~min}$. After washing away the excess dye equal number $\left(0.5 \times 10^{6}\right)$ of cells were distributed in a 96-well plate; $200 \mu \mathrm{M}$ NIC was added and 
ROS production was immediately determined as described elsewhere (15). ROS production was calculated as the increase in fluorescence $/ 30 \mathrm{~min} / 0.5 \times 10^{6}$ cells and expressed as a percentage of that of corresponding untreated cells.

Viability assay. For assessing cellular viability, the Cell Titer Blue fluorescent kit was used (Promega, Madison, WI, USA). Briefly, cells grown in 96-well plates were transfected/treated as needed and reagent added. After incubation for $2 \mathrm{~h}$ at $37^{\circ} \mathrm{C}$ fluorescence was determined. Results were expressed as percentage of control values

Reporter luciferase assay. NRK52E cells grown in 24-well plates were transfected with either a MnSOD-promoter-reporter-luciferase (16), a luciferase plasmid that harbors 6 canonical FOXO binding sites (6xDBE) to determine FOXO-dependent transcription (17) or cAMP responsive element (CRE) luciferase (Stratagene, La Jolla, CA, USA) together with Renilla luciferase (Promega, Madison, WI, USA) by using Lipofectamine 3000 reagent (Life Technologies, Grand Island, NY, USA). $24 \mathrm{~h}$ after treatment firefly and renilla luciferase activities were determined by using the Dual Luciferase assay kit (Promega, Madison, WI, USA). Luciferase activities were calculated as the ratio of the firefly and renilla activities and expressed as a percentage of the control (untreated) values.

Knockdown of FoxO3a and MnSOD. Knockdown of FoxO3a was performed via transfection of a FoxO3a siRNA (Cell Signaling Technologies, Danvers, MA, USA) using the Lipofectamine 3000 transfection reagent (Thermofisher Scientific, Waltham, MA, USA) according to the manufacturer's recommendation. To knockdown MnSOD, NRK52E cells were transfected with a short-hairpinMnSOD (shMnSOD) plasmid as described elsewhere (14). Knockdown of MnSOD was verified by Western blotting (14).

Statistical analysis. Continuous variables are expressed as means and standard deviations (S.D.). One-way ANOVA with Holm-Sidak post-hoc test was used to evaluate differences between groups. Differences between means were considered significant if $p<0.05$. All analyses were performed using the GraphPad InStat3 (La Jolla, CA, USA) software package.

\section{Results}

Resveratrol protects renal proximal tubule cells from nicotine-mediated oxidative stress and injury. NRK52E cells were treated with vehicle or $20 \mu \mathrm{M}$ RES overnight and $200 \mu \mathrm{M}$ NIC-mediated ROS production was determined as described in Materials and Methods. Figure 1A shows that pre-treatment with RES completely abolished NIC-induced ROS release. It is important to note that RES alone attenuated endogenous ROS production. Since NIC-induced ROS leads to significant cytotoxicity in renal proximal tubule cells (7), we determined whether RES protects cells from NIC-associated cytotoxicity. Accordingly, NRK52E cells were treated with vehicle or $20 \mu \mathrm{M}$ RES for $24 \mathrm{~h}$ followed by treatment with $200 \mu \mathrm{M}$ NIC. Viability of cells was determined $24 \mathrm{~h}$ later. Results shown in Figure 1B demonstrate that RES, indeed, protects from cytotoxicity imposed by NIC treatment.
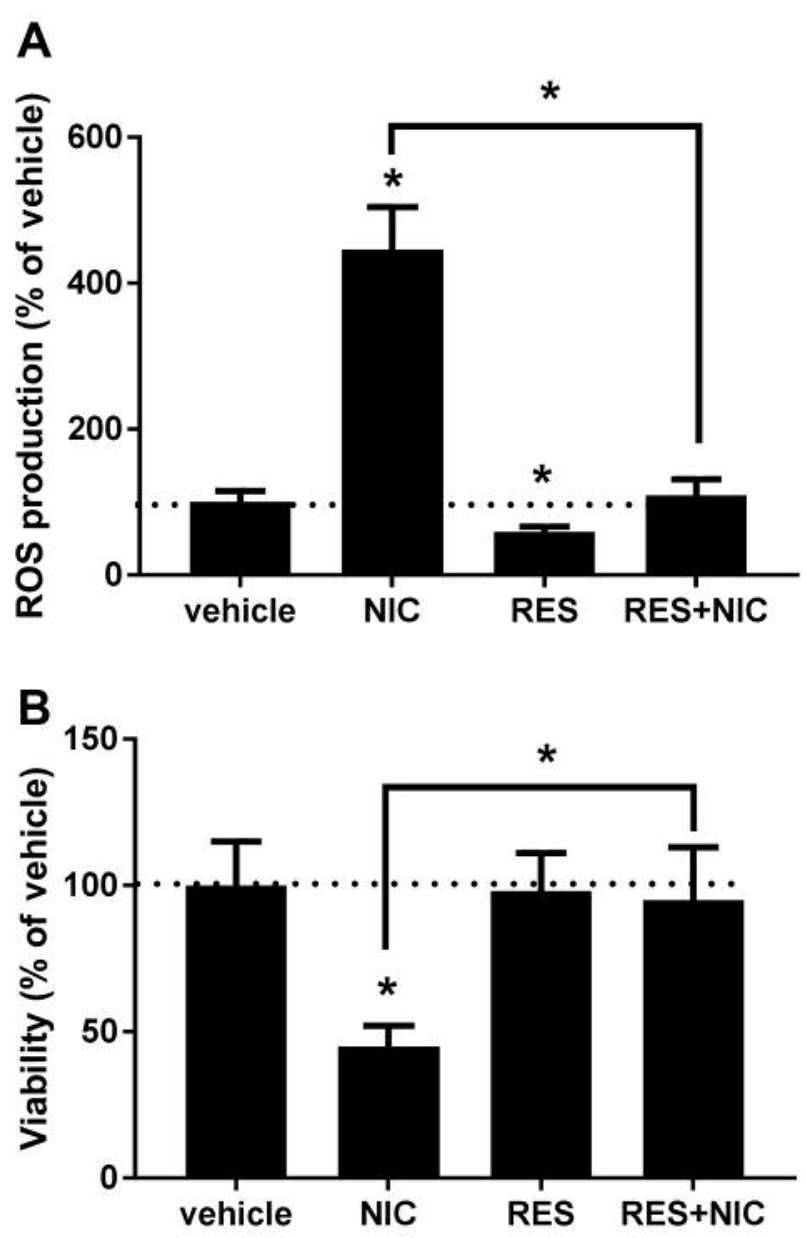

Figure 1. Resveratrol protects renal proximal tubule cells from nicotinemediated oxidative injury. NRK52E cells were pretreated with vehicle or $20 \mu M$ RES for $24 h$ and $200 \mu M$ NIC-induced ROS production (A) as well as viability $(B)$ was determined. $n=3 ; * p<0.05$ compared to vehicle or as indicated. The dotted line marks control (vehicle-treated) value.

Resveratrol activates the promoter of the antioxidant MnSOD gene via FoxO3a. To determine whether RES activates the antioxidant MnSOD, NRK52E cells were transfected with a reporter luciferase that harbors the promoter of the MnSOD gene (16) together with a renilla luciferase, as described in Materials and Methods. Transfected cells were then treated with $20 \mu \mathrm{M}$ RES and 24 $\mathrm{h}$ later luciferase activities were determined. Figure 2A shows that RES significantly activated the MnSOD promoter. To verify that RES induces the MnSOD promoter via CREB (13) or FoxO (18) transcription factors, NRK52E cells were transfected with either a CRE or a reporter luciferase plasmid that harbors 6 canonical FOXO binding sites $(6 \mathrm{xDBE})$ together with Renilla luciferase. Figure $2 \mathrm{~A}$ demonstrates that $24 \mathrm{~h}$ treatment with $20 \mu \mathrm{M}$ RES significantly increased the activity of $6 \mathrm{xDBE}$ reporter, but 


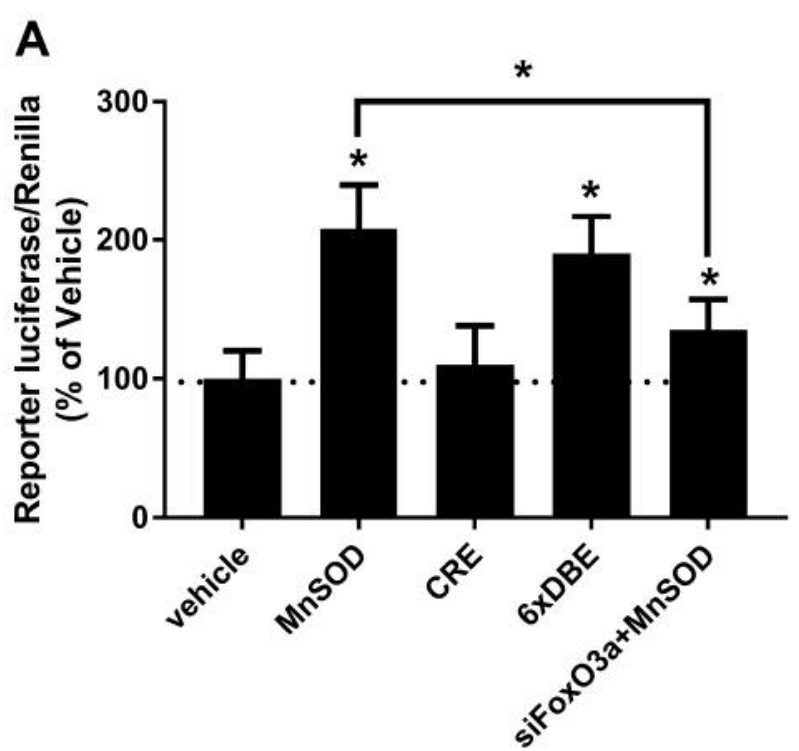

B

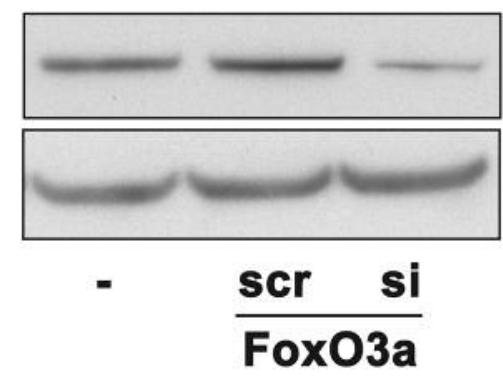

FoxO3a

\section{actin}

Figure 2. Resveratrol activates the promoter of the MnSOD gene via FoxO3a. (A) NRK52E cells were transfected with either a MnSOD promoter, CRE or 6xDBE luciferase reporter together with a renilla luciferase. Some cells were co-transfected with a FoxO3a siRNA plus MnSOD/renilla plasmids. Transfected cells were treated with $20 \mu \mathrm{M}$ RES for 24 h. $n=3 ; * p<0.05$ compared to vehicle or as indicated. The dotted line marks control (vehicle-treated) value. (B) The extent of knockdown was verified by Western blotting in NRK52E cells that were transfected with a FoxO3A siRNA (siFoxO3a). Data are representatives of 3 individual experiments. scrFoxO3a: scrambled FoxO3a.

not the CRE reporter. To demonstrate that FoxO plays a role in RES-mediated induction of the MnSOD promoter, NRK52E cells were co-transfected with a FoxO3a siRNA plus a MnSOD promoter luciferase and treated with $20 \mu \mathrm{M}$ RES for $24 \mathrm{~h}$. Figure 2A demonstrates that silencing FoxO3a (siFoxO3a) significantly attenuated RES-mediated induction of the MnSOD promoter. The extent of siRNA-mediated FoxO3a knockdown $(\sim 70 \%)$ is shown in Figure $2 \mathrm{~B}$.

Silencing of MnSOD attenuates the protective effects of resveratrol on nicotine-associated cytotoxicity. NRK52E

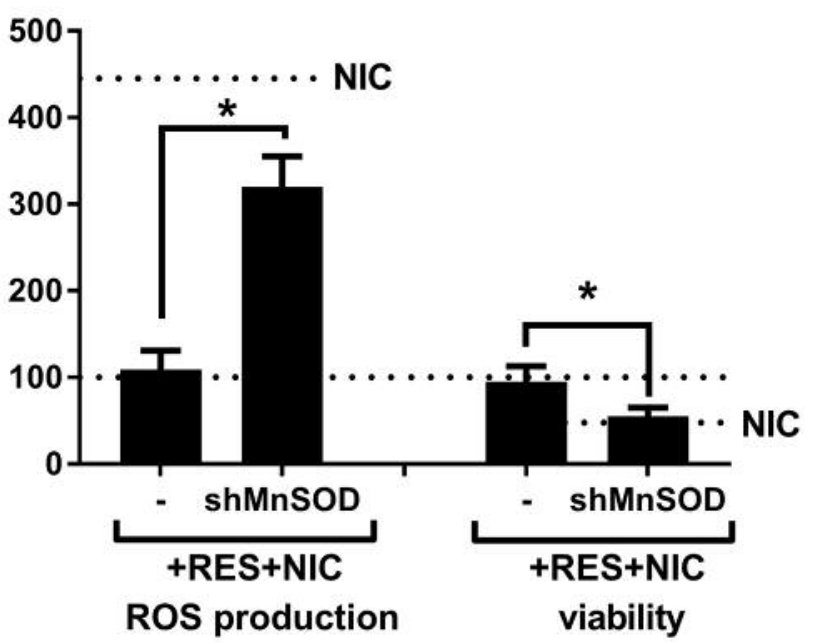

Figure 3. Resveratrol protects renal proximal tubule cells from nicotineinduced cytotoxicity via MnSOD. NRK52E cells were co-transfected with shMnSOD together with MnSOD promoter reporter and treated with $20 \mu M$ RES. After 24 h $200 \mu M$ NIC-induced ROS production and cell viability were determined as described in Materials and Methods. $n=3$; * $p<0.05$; upper and lower dotted lines mark NIC values for ROS and viability (from Figure 1), respectively.

cells were transfected with shMnSOD to knockdown MnSOD expression as described in Materials and Methods: extent of silencing was determined elsewhere (14). Control cells were transfected with an empty plasmid. Transfected cells were pretreated with $20 \mu \mathrm{M}$ RES overnight and $200 \mu \mathrm{M}$ NIC-mediated ROS production and cytotoxicity were determined. Figure 3 demonstrates that silencing of MnSOD reversed protective effects of RES on NIC-mediated ROS release. Not surprisingly, silencing of MnSOD also decreased viability of cells treated with RES+NIC.

\section{Discussion}

It is well accepted that smoking/NIC exposure increases oxidative stress in the kidney that enhances the risk for development and progression of renal chronic diseases (1, 68). NIC-associated oxidative stress is related to release of excessive amount of ROS from the mitochondria $(7,8,19)$, which may not be counterbalanced by the resident antioxidant system such as the mitochondrial MnSOD (20). Therefore, modalities that reinforce the endogenous antioxidant system would be beneficial in ameliorating smoking/NIC exposure-associated oxidative stress.

Resveratrol (RES) is a naturally occurring antioxidant, found in high concentration in grapes and wine (21). Importantly, RES activates mitochondrial antioxidant enzymes, such as MnSOD, that decrease mitochondrial ROS 
production (22). Thus, RES is a good candidate to prevent smoking-associated oxidative stress. Indeed, several studies have shown beneficial effects of RES on smoking in various organs (22-24), while the kidney is barely studied (10). Our study demonstrated that RES protected rat renal proximal tubule cells from NIC-mediated oxidative stress (Figure 1B) by reducing ROS production (Figure 1A). We also found that this protection is attributed to the activation of MnSOD (Figure 3).

Transcriptional regulation of MnSOD is associated with induction of several transcription factors that bind to the promoter of the MnSOD gene. One candidate is the CREB transcription factor (13), which is actually activated by RES in the heart (25) and brain (26). In our system, we did not find significant induction of a CRE luciferase reporter after treatment with RES (Figure 2). Besides CREB, FOXO transcription factors are also involved in activation of the MnSOD promoter (27). Renal proximal tubule cells express FoxO3a (28) and earlier we showed that FoxO3a is involved in MnSOD induction in renal proximal tubule cells (14). Studies have also shown that RES activates FoxO3a in kidney cells (11). Our study confirmed this hypothesis: RES treatment significantly induced FOXOdependent transcription (Figure 2). We also demonstrated that FoxO3a mediates RES-dependent induction of the MnSOD promoter (Figure 2).

In conclusion, RES attenuates NIC exposure-associated induction of ROS release and consequent renal toxicity via FoxO3a-dependent induction of the antioxidant MnSOD gene. Our results showed that treatment with RES may be used to ameliorate smoking/NIC exposure-inflicted renal risk.

\section{Acknowledgements}

This work was supported by a grant from the Department of Pediatrics at the University of Mississippi Medical Center and the Bower Foundation.

\section{References}

1 Jaimes EA, Tian RX and Raij L: Nicotine: The link between cigarette smoking and the progression of renal injury? Am $\mathrm{J}$ Physiol Heart Circ Physiol 292(1): H76-82, 2007.

2 Orth SR and Hallan SI: Smoking: A risk factor for progression of chronic kidney disease and for cardiovascular morbidity and mortality in renal patients - absence of evidence or evidence of absence? Clin J Am Soc Nephrol 3(1): 226-236, 2008.

3 Baeza-Loya S, Viswanath H, Carter A, Molfese DL, Velasquez KM, Baldwin PR, Thompson-Lake DG, Sharp C, Fowler JC, De La Garza R, 2nd and Salas R: Perceptions about E-cigarette safety may lead to e-smoking during pregnancy. Bull Menninger Clin 78(3): 243-252, 2014.

4 Callahan-Lyon P: Electronic cigarettes: Human health effects. Tob Control 23(Suppl 2): 36-40, 2014.
5 Chiolero A, Faeh D, Paccaud F and Cornuz J: Consequences of smoking for body weight, body fat distribution, and insulin resistance. Am J Clin Nutr 87(4): 801-809, 2008.

6 Orth SR, Viedt C and Ritz E: Adverse effects of smoking in the renal patient. Tohoku J Exp Med 194(1): 1-15, 2001.

7 Arany I, Grifoni S, Clark JS, Csongradi E, Maric C and Juncos LA: Chronic nicotine exposure exacerbates acute renal ischemic injury. Am J Physiol Renal Physiol 301(1): F125-133, 2011.

8 Arany I, Clark J, Reed DK and Juncos LA: Chronic nicotine exposure augments renal oxidative stress and injury through transcriptional activation of p66shc. Nephrol Dial Transplant 28(6): 1417-1425, 2013.

9 Rodrigo R and Bosco C: Oxidative stress and protective effects of polyphenols: Comparative studies in human and rodent kidney. A review. Comp Biochem Physiol C Toxicol Pharmacol 142(3-4): 317-327, 2006.

10 Kurus M, Ugras M and Esrefoglu M: Effect of resveratrol on tubular damage and interstitial fibrosis in kidneys of rats exposed to cigarette smoke. Toxicol Ind Health 25(8): 539-544, 2009.

11 Fu B, Zhao J, Peng W, Wu H and Zhang Y: Resveratrol rescues cadmium-induced mitochondrial injury by enhancing transcriptional regulation of PGC-1alpha and SOD2 via the SIRT3/FoxO3a pathway in TCMK-1 cells. Biochem Biophys Res Commun 486(1): 198-204, 2017.

12 Wang HJ, Wang Q, Lv ZM, Wang CL, Li CP and Rong YL: Resveratrol appears to protect against oxidative stress and steroidogenesis collapse in mice fed high-calorie and highcholesterol diet. Andrologia 47(1): 59-65, 2015.

13 Kim HP, Roe JH, Chock PB and Yim MB: Transcriptional activation of the human manganese superoxide dismutase gene mediated by tetradecanoylphorbol acetate. J Biol Chem 274(52): 37455-37460, 1999.

14 Arany I, Hall S, Reed DK and Dixit M: The pro-oxidant gene p66shc increases nicotine exposure-induced lipotoxic oxidative stress in renal proximal tubule cells. Mol Med Rep 14(3): 27712777, 2016.

15 Arany I, Faisal A, Clark JS, Vera T, Baliga R and Nagamine Y: p66shc-mediated mitochondrial dysfunction in renal proximal tubule cells during oxidative injury. Am J Physiol Renal Physiol 298(5): F1214-1221, 2010.

16 Essers MA, Weijzen S, de Vries-Smits AM, Saarloos I, de Ruiter ND, Bos JL and Burgering BM: Foxo transcription factor activation by oxidative stress mediated by the small GTPase Ral and JNK. EMBO J 23(24): 4802-4812, 2004.

17 Brenkman AB, van den Broek NJ, de Keizer PL, van Gent DC and Burgering BM: The DNA damage repair protein ku70 interacts with FoxO4 to coordinate a conserved cellular stress response. FASEB J 24(11): 4271-4280, 2010.

18 Kops GJ, Dansen TB, Polderman PE, Saarloos I, Wirtz KW, Coffer PJ, Huang TT, Bos JL, Medema RH and Burgering BM: Forkhead transcription factor FoxO3a protects quiescent cells from oxidative stress. Nature 419(6904): 316-321, 2002.

19 Cormier A, Morin C, Zini R, Tillement JP and Lagrue G: In vitro effects of nicotine on mitochondrial respiration and superoxide anion generation. Brain Res 900(1): 72-79, 2001.

20 Bruin JE, Petre MA, Lehman MA, Raha S, Gerstein HC, Morrison KM and Holloway AC: Maternal nicotine exposure increases oxidative stress in the offspring. Free Rad Biol Med 44(11): 1919-1925, 2008. 
21 Rabassa M, Zamora-Ros R, Urpi-Sarda M and Andres-Lacueva $\mathrm{C}$ : Resveratrol metabolite profiling in clinical nutrition research-from diet to uncovering disease risk biomarkers: Epidemiological evidence. Ann N Y Acad Sci 1348(1): 107-115, 2015.

22 Jardim FR, de Rossi FT, Nascimento MX, da Silva Barros RG, Borges PA, Prescilio IC and de Oliveira MR: Resveratrol and brain mitochondria: A review. Mol Neurobiol, 2017. doi: 10.1007/s12035-017-0448-z. [Epub ahead of print]

23 Sticozzi C, Belmonte G, Cervellati F, Muresan XM, Pessina F, Lim Y, Forman HJ and Valacchi G: Resveratrol protects sr-b1 levels in keratinocytes exposed to cigarette smoke. Free Radic Biol Med 69: 50-57, 2014

24 Kurus M, Firat Y, Cetin A, Kelles M and Otlu A: The effect of resveratrol in tracheal tissue of rats exposed to cigarette smoke. Inhal Toxicol 21(12): 979-984, 2009.

25 Das S, Cordis GA, Maulik N and Das DK: Pharmacological preconditioning with resveratrol: Role of creb-dependent bcl-2 signaling via adenosine A3 receptor activation. Am J Physiol Heart Circ Physiol 288(1): H328-335, 2005.
26 Thiel $G$ and Rossler OG: Resveratrol regulates gene transcription via activation of stimulus-responsive transcription factors. Pharmacol Res 117: 166-176, 2017.

27 Charitou P and Burgering BM: Forkhead box(o) in control of reactive oxygen species and genomic stability to ensure healthy lifespan. Antioxid Redox Signal 19(12): 1400-1419, 2013.

28 Fujiki K, Inamura $\mathrm{H}$ and Matsuoka M: Phosphorylation of FoxO3a by PI3K/AKT pathway in HK-2 renal proximal tubular epithelial cells exposed to cadmium. Arch Toxicol 87(12): 2119$2127,2013$.
Received March 23, 2017

Revised April 6, 2017

Accepted April 7, 2017 